\title{
Self-organizing photorefractive frequency demultiplexer
}

\author{
Mark Saffman, Claus Benkert, and Dana Z. Anderson
}

Department of Physics and Joint Institute for Laboratory Astrophysics, University of Colorado, Boulder, Colorado 80309-0440

Received July 8, 1991

\begin{abstract}
We demonstrate a self-organizing photorefractive circuit that demultiplexes a beam that has two signals imposed on separate optical carrier frequencies into two beams, each containing one of the signals on its carrier. Unlike conventional demultiplexing techniques, this method requires little a priori knowledge about the carrier frequencies. The signal channels must be spatially uncorrelated, and their frequency separation must be more than the photorefractive response bandwidth (hertz to kilohertz). The optical circuit uses no local oscillator, and the photorefractive response bandwidth places no upper bound on the channel bandwidth. Experimental results for demultiplexing a beam that has two signals, with a $\mathrm{BaTiO}_{3}$ circuit, show contrast ratios of better than $40: 1$ at the outputs.
\end{abstract}

Consider the task of demultiplexing a number of signals carried on a multimode fiber, and assume that we have no a priori information about the carrier frequencies other than that they are different. How can the channels at the output of the fiber be separated? One might use a grating, but then the carriers would need to be well separated in wavelength, by at least an angstrom or so, and we do not know ahead of time that they are. One might use interferometric techniques, but these require additional frequency scan and locking electronics that must also avoid possible degeneracies in resonance conditions. To make things worse, neither approach can handle the high spatial content of the speckle patterns in a straightforward manner.

Our a priori information about the signals is limited to the fact that they have different frequencies, and, in the case of a highly multimode fiber, that the individual speckle patterns of the signals will be uncorrelated. Using this information alone, and drawing on some elementary concepts from neural networks, we have constructed a photorefractive optical circuit that self-organizes to demultiplex incoming optical signals according to their temporal and spatial differences.

An optical circuit that demultiplexes two signal channels is shown schematically in Fig. 1. The self-organizing behavior ${ }^{1}$ arises from competitive interactions in multimode photorefractive ring resonators. ${ }^{2,3}$ The combination of the competitive interaction between resonators in the gain medium and a local interaction in each resonator, which we refer to as reflexive gain, leads to a steady-state operating condition in which each resonator chooses to oscillate at one of the pump frequencies. A portion of the oscillating energy is then coupled out of the resonators to give the demultiplexed outputs. Note that the optical circuit is purely signal driven: there is no local oscillator, and there is no local power source.

Arguments based on steady-state energy transfer in photorefractive two-beam coupling will clarify the operation of the circuit. We will, for simplicity, con- sider a two-resonator circuit demultiplexing a twosignal input beam. To demultiplex $N$ frequencies we would include $N$ resonators, each sharing the same pump volume, and each with a reflexive photorefractive interaction. The resonators support many transverse modes, and to simplify the analysis we assume that the oscillating-mode superposition is always on resonance, so pump-detuning effects need not be considered. When the circuit is above threshold the two rings compete for energy in the shared gain medium. Without additional interactions the rings are at most neutrally coupled, ${ }^{4}$ and any mixture of the incident frequencies can oscillate in each ring.

The key to achieving the demultiplexing behavior is a nonlinearity that raises the cavity losses when two signals try to oscillate in the same ring. There are two distinct cases to be considered. In the first case the two signals may attempt to oscillate with identical spatial-mode superpositions. This is a high-loss configuration since the oscillating signals are now spatially matched, and each one will scatter off the grating owing to the interference of the other oscillating signal and its pump. Thus one spatialmode superposition oscillating at two frequencies is disfavored. In the second case, where the two signals choose orthogonal spatial-mode superpositions, the desired nonlinearity is provided by the reflexive gain interaction. A portion of the oscillating energy is taken out of each ring and then coupled back into the same ring by photorefractive two-beam coupling, as is detailed in Fig. 2: The standard equations for photorefractive two-beam coupling ${ }^{5,6}$ can be used to derive an expression for the steadystate transmission $T$ of the resonator beam through the reflexive gain interaction. If only one signal oscillates in the resonator beam we find that $T=$ $1 /\left[1+r \exp \left(-G_{R}\right)\right]$ in the absence of reflective and absorptive losses. The pump-to-signal ratio incident upon the photorefractive medium is given by $r=\delta /(1-\delta)$, where $\delta$ is the intensity reflection coefficient of the beam splitter, $\exp \left(G_{R}\right)$ is the smallsignal gain in the photorefractive medium, and 


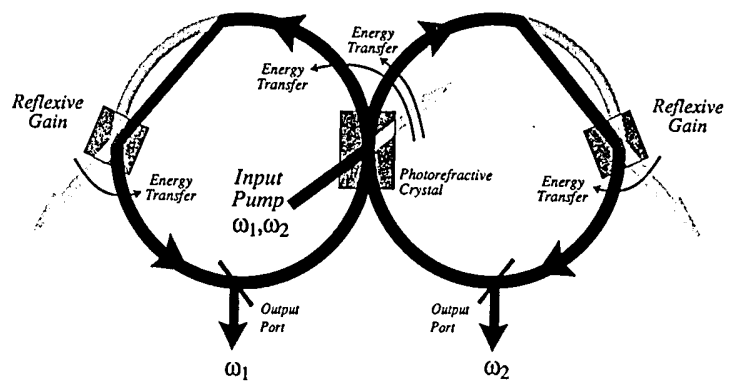

Fig. 1. Schematic diagram of the self-organizing circuit.

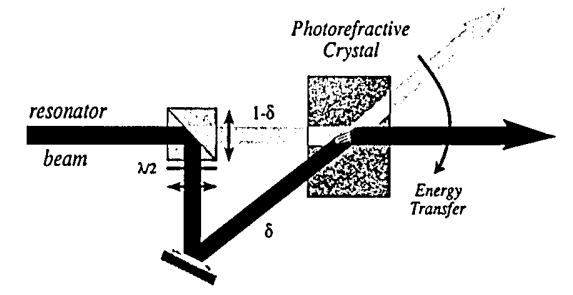

Fig. 2. Reflexive gain interaction. A fraction $\delta$ of the incident intensity is removed from the resonator beam and then coupled in again by photorefractive two-wave mixing.

$G_{R}=\Gamma_{R} l_{R}$, with $\Gamma_{R}$ the photorefractive coupling constant and $l_{R}$ the interaction length. In the steady state the reflexive interaction simply contributes to the passive resonator losses. ${ }^{7}$ When two signals, each with orthogonal transverse-mode superpositions and different carrier frequencies, oscillate in one ring, two orthogonal gratings build up in the reflexive gain crystal. The effective steady-state coupling strength between two waves that have the same frequency when several mutually incoherent beams are present can be written as $G_{R \text {,eff }}=$ $G_{R}\left(I_{\text {int }} / I\right)$, where $I$ is the total intensity and $I_{\text {int }}$ is the portion of the intensity that writes the photorefractive grating. Therefore, when two frequencies are present, the effective coupling for each mode superposition is reduced from the single-frequency case. $T$ is correspondingly reduced, hence multifrequency oscillation is again disfavored.

The reflexive gain interaction also serves to ensure a high contrast ratio between the signal intensities in each ring. Even when the input signals are spatially uncorrelated, fanning gratings in the gain medium will couple both input signals to both resonator rings, which tends to reduce the oscillating contrast ratio. Nonetheless, a high steady-state contrast ratio can be achieved because the reflexive gain interaction actively enhances the contrast ratio, counteracting the contrast ratio reduction in the gain medium.

The contrast ratio enhancement in the reflexive gain interaction can be calculated as follows. Let the oscillating beam consist of two fields, having different frequencies and occupying orthogonal mode superpositions, with intensities $I_{1}$ and $I_{2}$. The contrast ratio at the input to the reflexive gain interaction is defined as $q=I_{1} / I_{2}$. The effective steady-state coupling strengths for the two signals are $G_{R 1, \text { off }}=G_{R} q /(1+q)$ and $G_{R 2, \text { off }}=G_{R} 1 /(1+q)$, and the contrast ratio at the output of the reflexive gain interaction is then given by

$$
\tilde{q}=q \frac{1+r \exp \left(-G_{R} \frac{1}{1+q}\right)}{1+r \exp \left(-G_{R} \frac{q}{1+q}\right)}
$$

The contrast ratio enhancement follows, because for $q>1, \tilde{q}>q$, and for $q<1, \tilde{q}<q$.

We have attempted to provide some heuristic arguments for the observed behavior of the demultiplexer. It is also useful to know the values of circuit parameters that are necessary to ensure the desired behavior. In order to demultiplex $N$ signals there must be sufficient gain for the resonators to be above threshold. The oscillation threshold may be derived from small-signal gain considerations analogous to those for a single-crystal photorefractive resonator. ${ }^{8}$ For an $N$-ring circuit pumped by a beam having $N$ signals of equal intensity we must have

$$
(1-L) \frac{1}{1+r \exp \left(-G_{R} / N\right)} \exp \left(G_{P} / N\right)>1,
$$

where $L$ is the passive cavity loss. The conditions that ensure stability of the demultiplexing state to small perturbations, and the instability of all other steady-state conditions, have yet to be determined.

We have experimentally demonstrated the circuit described above as shown in Fig. 3. The output from an Ar-ion laser operating at $514.5 \mathrm{~nm}$ is split into two and then frequency shifted with acoustooptic cells to generate two beams with a frequency separation of $280 \mathrm{MHz}$. The beams are then coupled into a 1.5-m-long step-index, $100-\mu \mathrm{m}$-core, multimode fiber. By arranging each beam to have a different angle of incidence on the fiber end face, we obtain uncorrelated output speckle patterns, even with a relatively short fiber. ${ }^{9}$ The output of the fiber is then used to pump the photorefractive resonators. By modulating the acousto-optic cells with low-frequency square waves, the carrier content in

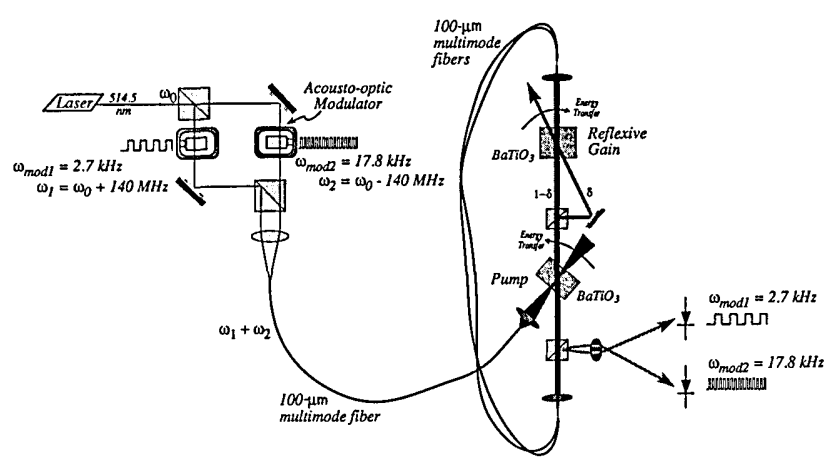

Fig. 3. Experimental demonstration of a self-organizing frequency demultiplexer. The circuit configuration is equivalent to that shown in Fig. 1. Each resonator ring has a length of $\sim 2 \mathrm{~m}$, of which $1 \mathrm{~m}$ is $100-\mu \mathrm{m}$-core multimode fiber. The two resonator rings are positioned on top of each other with the modes crossing in the pump crystal and occupying separate volumes in the reflexive gain crystal. The pump crystal is $45^{\circ}$-cut $\mathrm{BaTiO}_{3}$, and the reflexive gain crystal is $0^{\circ}$-cut $\mathrm{BaTiO}_{3}$. The resonator parameters are $\exp \left(G_{P}\right)=1700, \exp \left(G_{R}\right)=230,1-L=$ 0.01 , and $\delta=0.96$. 


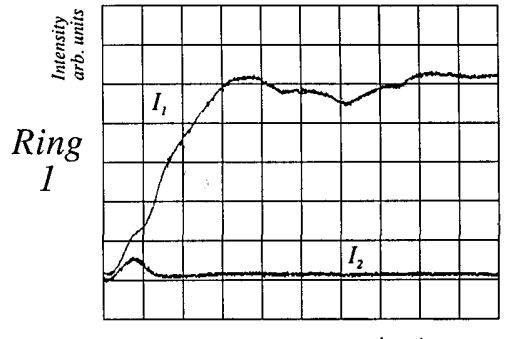

$\rightarrow|10 s| \leftarrow$

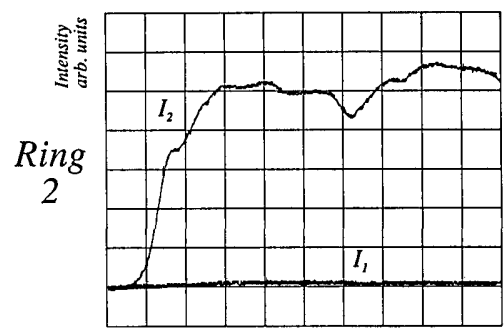

Fig. 4. Transient behavior of the circuit. The curves represent the envelopes of the square waves driving the acousto-optic modulators. Signal $I_{1}$ on carrier $\omega_{1}$ oscillates in resonator ring 1 , and signal $I_{2}$ on carrier $\omega_{2}$ oscillates in resonator ring 2 .

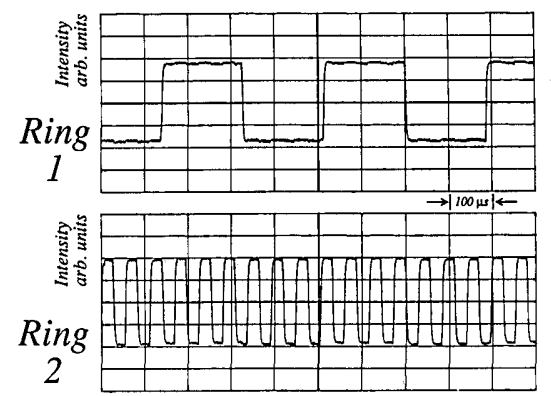

Fig. 5. Outputs of the two resonator rings. The signal carrier frequencies are separated by $280 \mathrm{MHz}$.

each ring can be monitored by detecting the output intensities with low-speed photodiodes.

When the input signals are presented to the circuit it takes a few seconds for the self-organization process to reach steady state. The mapping of the input signals into resonator modes is initially noise driven, so which signal will oscillate in which ring is random. We observe the dynamical evolution by detecting the envelopes of the carrier modulations, as shown in Fig. 4. With a total pump intensity of $10 \mathrm{~mW}$, focused to a $0.58-\mathrm{mm}$-diameter spot in the pump crystal, the circuit takes approximately $10 \mathrm{~s}$ to reach the high-contrast-ratio demultiplexing state shown in Fig. 5. This operating state is then stable for as long as the pump beam is present. The total steady-state oscillating intensity in the two rings reached $0.4 \%$ of the pump intensity. Measurements with a spectrum analyzer show an output contrast ratio of better than 40:1 in each ring. This is consistent with our previous holographic measurements of the orthogonality of speckle patterns transmitted over a short length of fiber, ${ }^{9}$ where we found bestcase cross-talk levels of 50:1. In order for the circuit to demultiplex the input signals, their intensities must be matched to within approximately
$10 \%$, otherwise the stronger signal will oscillate in both rings. We are currently investigating alternative designs that may relax this constraint.

This circuit has potential application to optical communications systems. We showed recently that the spatial-mode superposition can be used as a multiplex parameter to transmit multiple communication channels on a single multimode fiber. ${ }^{9}$ In that case it was necessary to record, and periodically refresh, the holograms that demultiplexed the channels. The circuit described here demultiplexes spatially distinct channels while continuously adapting to slow variations in the spatial-mode superposition owing to drifts in the carrier frequency or perturbations to the fiber. The circuit can also be used with channels that have been transmitted on a single-mode fiber. Taking the output of the single mode fiber and sending it through a sufficiently long length of multimode fiber, before pumping the self-organizing circuit, will impress a distinct spatial mode on each channel..$^{10}$

In the parlance of neural networks this circuit demultiplexes through a self-organizing process. The circuit learns to associate distinct input signals with different resonator modes. The essential characteristics of the input signals that allow them to be separated are their spatial and temporal orthogonality. We have used acousto-optic modulators to generate well-defined input signals. In principle any type of transduction mechanism that provides distinct input frequencies with spatially distinct representations could be used with this circuit.

We are grateful for the support of the U.S. Air Force Office of Scientific Research through contract 90-0198. Mark Saffman acknowledges support provided by a U.S. Air Force Office of Scientific Research laboratory graduate fellowship.

\section{References}

1. T. Kohonen, Self-Organization and Associative Memory, 2nd ed. (Springer-Verlag, Berlin, 1989).

2. D. Z. Anderson, C. Benkert, B. Chorbajian, and A. Hermanns, Opt. Lett. 16, 250 (1991).

3. C. Benkert and D. Z. Anderson, Phys. Rev. A 44, 4633 (1991).

4. D. Z. Anderson and R. Saxena, J. Opt. Soc. Am. B 4, 164 (1987).

5. N. V. Kukhtarev, V. B. Markov, S. G. Odulov, M. S. Soskin, and V. L. Vinetskii, Ferroelectrics 22, 949 (1979).

6. N. V. Kukhtarev, V. B. Markov, S. G. Odulov, M. S. Soskin, and V. L. Vinetskii, Ferroelectrics 22, 961 (1979).

7. Interestingly, when the pump consists of a single frequency the influence of the reflexive gain interaction on the dynamical evolution of the circuit leads to a flip-flop behavior between the rings, equivalent to that reported in Ref. 2.

8. J. O. White, M. Cronin-Golomb, B. Fischer, and A. Yariv, Appl. Phys. Lett. 40, 450 (1982).

9. M. Saffman and D. Z. Anderson, Opt. Lett. 16, 300 (1991).

10. M. Koga and T. Matsumoto, IEEE Photon. Technol. Lett. 2, 487 (1990). 